\title{
Review
}

\section{Innervation of the Smooth Muscle of the Lower Urinary Tract}

\author{
Kate E CREED \\ School of Veterinary Studies, Murdoch University, Western Australia 6150 \\ (Soc Sm Musc Res Japan Yamaguchi August 1994)
}

\begin{abstract}
The lower urinary tract in mammals consists of the bladder, a storage organ for urine, and the urethra through which urine is expelled to the outside. In the male the urethra extends outside the body as the penis.

The lower urinary tract is innervated by pelvic (parasympathetic) nerves and hypogastric nerves which combine on each side at the pelvic plexus. Fibres pass to the bladder, urethra, genital organs, rectum and blood vessels. In addition to the pelvic and hypogastric nerves there is sacral outflow to skeletal muscle, such as the external sphincters of the urethra and anus, in the pudendal nerve and the sympathetic chain extends caudally with branches lying in the sacral outflow that supply the penis and other organs though not the bladder. Bladder evacuation and penile erection are produced by activity of the pelvic nerves whereas sympathetic activity usually dominates during urine storage and penile flaccidity, though it may not be essential for either.
\end{abstract}

\section{Parasympathetic innervation of the bladder}

The main parasympathetic input, in the pelvic nerves, uses at least 2 transmitters at both the bladder and penis. 100 years ago Langley showed that in many species the response of the bladder to pelvic nerve stimulation is partially atropine resistant both in vitro and in vivo. I have used several techniques to study the innervation. Mechanical recording in an organ bath of strips of detrusor from many species shows that nerve stimulation at $10 \mathrm{~Hz}$ produces a prolonged contraction. In the presence of atropine only a smaller, transient contraction occurs. Records of the effect of atropine on strips of guinea pig and rabbit detrusor muscle show that responses to all frequencies from 1 to $50 \mathrm{~Hz}$ are reduced. This was also seen in human strips but there is some controversy in the literature about the atropine resistance of human bladder strips. There has not been a detailed study in relation to clinical conditions but it is suggested that in healthy bladders atropine will abolish the entire response but the atropine resistance appears in certain compromised bladders.

More detail of these responses can be seen with the double sucrose gap, which records electrical and mechanical activity synchronously. In the rabbit a single stimulus to the nerves produces 2 distinct responses 1) an excitatory junction potential with superimposed spike and 2) a slow depolarization. The second part of the electrical response is selectively blocked by 
atropine and enhanced by eserine and is therefore due to acetylcholine. It should be noted that there is no evidence for an inhibitory response in the bladder from either electrical or mechanical recording.

Even though the 2nd, atropine sensitive response is small, it is responsible for a considerable contraction. Recordings with micro-electrodes show that acetylcholine can produce a considerable increase in frequency of action potentials with only a slight depolarization.

\section{Nature of second (rapid) transmitter}

Experiments with blocking drugs have suggested that the atropine resistant component is not due to amines. Peptides have been identified in the nerves but are unlikely to produce such a rapid response. ATP is a likely candidate: in the rabbit the analogue $\alpha \beta$ methylene ATP reduces only the first electrical response and the contraction. Similar results are obtained with strips from guinea pigs and other animals. However ATP only produces contraction when applied at high concentrations and the response is not selectively blocked by suramin, a drug that is reported to block the actions of $\mathrm{ATP}$ at $\mathrm{P} 2 \mathrm{x}$ receptors. In rabbit strips the responses are abolished by suramin, in guinea pig there is partial reduction and in sheep no effect.

In most species, therefore, pelvic nerve stimulation can increase bladder pressure through at least 2 neurotransmitters - one, possibly ATP, produces a rapid large contraction and the other, acetylcholine, produces a sustained increase in tone. The source of $\mathrm{Ca}^{2+}$ for contraction following action of transmitters has been investigated by recording channels in the membrane, concentrations of $\mathrm{IP}_{3}$, and dependence on extracellular $\mathrm{Ca}^{2+}$. In the bladder it was concluded that ATP opens channels in the membrane; acetylcholine releases the second messenger $\mathrm{IP}_{3}$ which affects intracellular stores, probably in the sarcoplasmic reticulum.

\section{Sympathetic innervation}

The sympathetic actions on the lower urinary tract can be explained by the presence of adrenergic nerves, though a small non-adrenergic component may also be present. The major sympathetic input to the bladder is in the hypogastric nerve and 3 sites of action have been identified. 1 /constriction of the urethra 2 /relaxation of the bladder detrusor muscle and $3 /$ inhibition of transmission at the pelvic plexus. The urethra receives dense adrenergic innervation and stimulation of the hypogastric nerves produces a strong constriction that is almost completely blocked by phentolamine. The absence of oestrogens decreases the affinity of the $\alpha$-adrenoceptors and may lead to incontinence. The bladder innervation, however, is sparse and high concentrations of noradrenaline are required to produce relaxation and decreased frequency of action potentials via $\beta$-adrenoceptors. The role of the pelvic plexus is incompletely understood. It has been shown that in some ganglion cells electrical activity can be induced by stimulation of hypogastric nerves and in some, hypogastric nerve stimulation reduces spike activity evoked in ganglion cells by simultaneous or subsequent pelvic nerve stimulation. However the distribution of these cells is not well understood nor are the details of the interaction between these two nerve inputs. 


\section{The urethra}

Compared with the bladder, little work has been done on the smooth muscle of the urethra and associated structures such as the prostate gland. Contraction by adrenergic sympathetic nerves are important for continence but during micturition its relaxation may not be necessary so long as bladder pressure exceeds urethral pressure. However, unlike the bladder, inhibitory responses can be recorded from the urethra. Of the 2 putative excitatory transmitters to the bladder, acetylcholine produces contraction of the urethra and ATP relaxation but cholinergic nerves are sparse.

In the female urethra there is no anatomical sphincter and closure involves the whole length and may be due to elastic properties as well as to contraction of the smooth muscle. Strips of muscle from the female urethra respond by contraction to nerve stimulation in the organ bath. Phentolamine, which blocks $\alpha$ adrenergic effects reduces the response. The inhibitory innervation shows up in the presence of phentolamine so that a relaxation now occurs on field stimulation. If the muscle tone is raised the response is almost always relaxation and this is reduced by the drug L-NAME indicating that nitric oxide is responsible. Similar results have been obtained for sheep, rabbits and dogs but not for guinea pigs. In the male the urethra is longer and, although there is greater thickening at the bladder neck, this is mostly due to the presence of connective tissue. Contraction of this region acts in part to prevent semen reflux into the bladder during ejaculation. In the dog the responses of the bladder neck and of the prostate capsule are similar to those seen in the female though the responses of the preprostatic urethra tend to be weaker. The prostate capsule also has nitric oxide inhibition (L-NAME) and in both the rabbit and dog there is both cholinergic and adrenergic excitatory action but the function is not understood. More detailed experiments must be carried out to identify other transmitters and to find out what the situation is with human urethra.

\section{The penis}

Transmission in the penis also involves more than one transmitter. Penile erection is induced by pelvic nerve stimulation and it is commonly accepted that it involves arterial vasodilation so that more blood enters the penis. Atropine reduces flow of blood from the penis in the veins but erection and stiffening of the penis still occurs. Pressure records of 3 chambers shows that they respond differently. This is now known to be because cholinergic nerves innervate the single corpus spongiosum which surrounds the urethra and dilation of the arterioles increases overall blood flow and penile volume. Pelvic nerve stimulation also increases blood flow into the paired corpora cavernosa but these form a closed system and pressure increases to systemic blood-pressure producing rigidity. Here however the transmission is resistant to atropine. Noradrenaline and ATP can also be excluded. There has been some support for VIP which is found in the effluent blood on pelvic nerve stimulation. The most likely candidate causing cavernosal dilation now seems to be nitric oxide, in human and animal preparations. The mechanism for its release is not yet understood.

The hypogastric nerve is not the major sympathetic input to the penis and its role seems 
to vary with species and individuals. Stimulation may enhance (some cats) or inhibit (some monkeys) erections or it may have no obvious effect (some dogs). The sympathetic fibres supplying the penis exit from the lumbar sympathetic chain. Stimulation of these prevents erections or reverse erections already induced by pelvic nerve stimulation. Noradrenaline is often injected into the penis to produce detumescence in priapism. The use of blocking agents in vivo or investigations of erectile tissue in vitro suggests that the inhibition can be explained by $\alpha 1$-adrenergic vasoconstriction in dogs but $\alpha 2$-receptors may be involved in other species since yohimbine is widely believed to be an aphrodisiac.

It is difficult to identify the exact site of action of transmitters. Does the smooth muscle within the corpora act in a similar way to the various arterioles that bring blood in? Are the veins actively closed or passively squeezed as the pressure increases? No anatomical structures responsible for this have been identified. The intricate relationship between vascular and support structures makes it difficult to isolate tissues for investigation.

\section{External influences}

Other factors may influence the action of transmitters, especially substances circulating in the blood or released by nerves. For instance prostaglandins at $10^{-6} \mathrm{M}$ increase contraction of bladder strips produced by acetylcholine or nerve stimulation but do not have an effect themselves at this concentration. Peptides, released by autonomic nerves, are not believed to act as transmitters but may have an overall effect on tone or alter responses to nerve stimulation. Oestrogen increases the sensitivity of the urethra to noradrenaline and alters the pattern of spontaneous electrical and mechanical activity.

Overall much work is still necessary to understand how nerves control the smooth muscles of the lower urinary tract. In particular studies must be carried out on transmission at the pelvic plexus and the action of neurotransmitters at the cellular level. During the course of this work it has become apparent that there is considerable species variation-perhaps remarkable when you consider that the bladder is only a reservoir and the penis a stiffened tube until you consider the varied life-styles and behavioural characteristics of mammals.

\section{References}

Creed, K.E., Carati, C.J. and Keogh, E.J. (1991). The physiology of penile erection. In: Oxford reviews of reproductive biology, ed. by S. Milligan, Oxford University Press, Oxford, vol. 13 p.p. 73-95.

Creed, K.E. (1994). Urinary tract. In : Pharmacology of Smooth Muscle, ed, by L. Szekeres and J. Gy. Papp, Handbook of Expt Pharm, Springer Verlag, Berlin/Heidelberg, vol. 111, p.p. 575-591. 